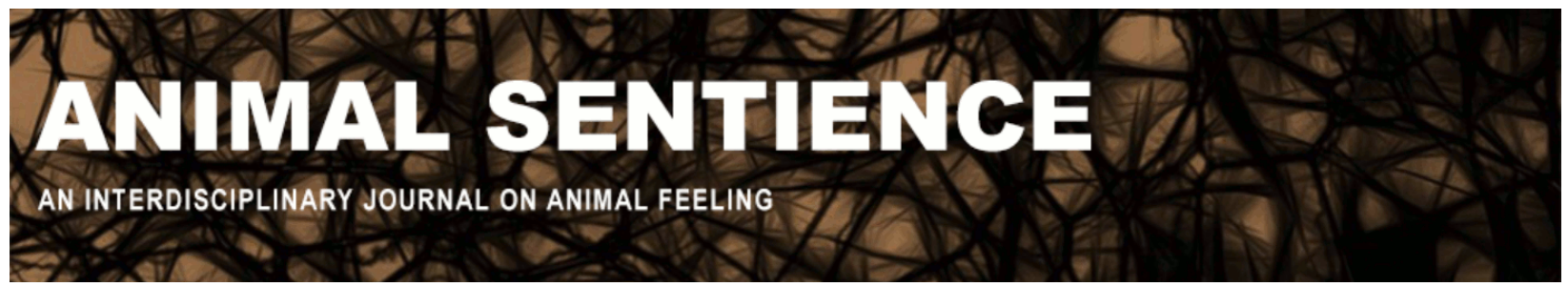

Mallatt, Jon and Feinberg, Todd E. (2020) Sentience in evolutionary context. Animal Sentience 29(14)

DOI: $10.51291 / 2377-7478.1599$

Date of submission: $2020-06-25$

Date of acceptance: 2020-06-28

(c)

This article has appeared in the journal Animal

Sentience, a peer-reviewed journal on animal

cognition and feeling. It has been made open access,

free for all, by WellBeing International and deposited

in the WBI Studies Repository. For more information,

please contact

wbisr-info@wellbeingintl.org.

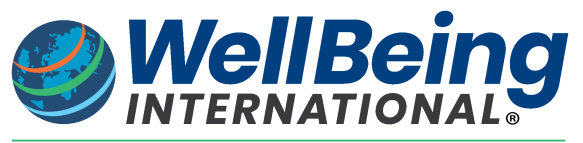

SOLUTIONS FOR PEOPLE, ANIMALS AND ENVIRONMENT 


\title{
Sentience in evolutionary context
}

Commentary on Mikhalevich \& Powell on Invertebrate Minds

\author{
Jon Mallatt \\ WWAMI Medical Education Program at The University of Idaho \\ Todd E. Feinberg \\ Psychiatry and Neurology Icahn School of Medicine at Mount Sinai
}

\begin{abstract}
We appreciate the goals of Mikhalevich \& Powell (M\&P) and largely agree with their conclusions but we differ on some of their definitions and terms. Affects (emotional feelings) should be part of sentience. Although the evidence presented for insect sentience is strong, we list some of the counterevidence that should be considered. Our own research supports M\&P's choice of arthropods, cephalopods, and vertebrates as the only sentient organisms with moral status.
\end{abstract}

Jon Mallatt, Clinical Associate Professor, WWAMI Medical Education Program, University of Idaho, does research on the origin of the major animal groups, especially vertebrates, and on the evolution and nature of consciousness. Website

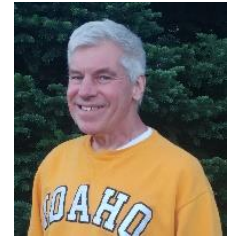

Todd E. Feinberg, Clinical Professor of Psychiatry and Neurology, Icahn School of Medicine at Mount Sinai does research on how the neurobiology of the brain creates consciousness and the individual's sense of identity. Website

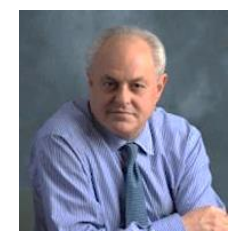

Mikhalevich \& Powell (2020) (M\&P) make a good argument that, for consistency, the same standards should be used to judge sentience and moral worth in vertebrates and invertebrates. Also valuable is how M\&P assemble a large amount of literature on invertebrate (mostly insect) sentience and cognition. We especially appreciate their pointing out that a capacity for pain is used too often as the sole standard for judging whether an animal is sentient, when many other affective states are possible (e.g., stress, starvation, pleasure) and should all be considered. Two of the commentaries to date, however, have centered on pain despite M\&P's plea against it (Elwood, 2020; Veit \& Huebner, 2020). Although we agree with many of M\&P's proposals and conclusions, we suggest that some of their positions should be further clarified.

1. Terminology and definitions. M\&P do not always make it clear on what basis they judge an animal to deserve moral status. In various places in the target article, the basis is said to be having mental states, cognition, sentience, cognition plus sentience, flexible behaviors, or affective states along with cognition. Most of these traits overlap, but some are fairly distinct (e.g., sentience and cognition), so it is difficult to know what the authors mean. Later in the text, 
however, they definitively settle on sentience as their basis for moral status, eliminating cognition because computers have cognitive functions. Sentience is the correct choice, and most of the other commentators understood that M\&P were using it (Balcombe, 2020; Browning and Veit, 2020; Cammaerts, 2020; Elwood, 2020; Figdor, 2020; Levy, 2020; Soryl, 2020). We just wish M\&P had established their sentience standard at the start of the target article.

Many different terms besides "sentience" are used to designate the most basic, minimal form of "consciousness" (yet another term). M\&P sometimes use these multiple terms in confusing ways. Table 1 is our version of the best usage, developed from our work on consciousness (Feinberg and Mallatt, 2018, 2019, 2020). Most other sources agree with us, especially that the basic type is called phenomenal consciousness and that this includes both images and affects (Block, 1995; Mellor, 2019), but M\&P do not.

\section{Table 1. Proposed terminology for basic, minimal consciousness}

\begin{tabular}{|cc|}
\hline \multicolumn{2}{|c|}{$\begin{array}{l}\text { Sentience } \\
\text { and consists of two kinds of experiences: }\end{array}$} \\
\hline A. & sensory experiences (including "images") \\
\hline B. & affective experiences (emotions and moods)*
\end{tabular}

*We assume all affects are conscious. For another view, see Adolphs and Anderson (2018).

A large part of the confusion comes from the way M\&P use the term "phenomenal consciousness." They point out that it is a difficult and controversial term. Then they say it is doubly "inadequate." From this, the reader might infer that they are rejecting it; but instead of rejecting it, they use phenomenal consciousness repeatedly without explicitly stating what they take it to mean (although the context implies that what they mean is "being capable of experience").

What is going on here? The only thing that is clear is that M\&P do not believe that phenomenal consciousness includes affect - that it is only a "precondition" for affect. According to other authors, however, when phenomenal consciousness is stripped of its philosophical complications, it includes experiencing affects as well as sensations (Block, 1995). Even Carruthers's deeply philosophical and elaborated analysis (to which M\&P refer) states that affect (valence) is in phenomenal consciousness (Carruthers, 2018, p. 676), not a separate thing that is glossed onto it, as M\&P seem to think.

We would accordingly recommend that M\&P include affect in their definition of phenomenal consciousness. This will avoid errors and confusion; then they can say that phenomenal consciousness, sentience, and experiencing are all equivalent (Table 1 ) and that that is what imparts moral worth. M\&P also use the key term "sentience" inconsistently. They first define it as the capacity for subjective experience, but later define it differently, as affect:

"Phenomenal consciousness alone is not an adequate basis by which to establish the existence of a welfare ... if things are to matter to an organism, it must be capable of experiencing states of affairs as pleasurable or aversive.... We think this affective glossing of conscious experience is better captured by the term sentience." 
The context of the target article indicates that M\&P only believe in the first definition, but the inconsistency adds to the reader's confusion. As M\&P's argument now stands, the semantics lead to a logical incoherence: Their current claims are that sentience is experience, that phenomenal consciousness is experience, that affects are different from phenomenal consciousness, and that sentience includes or even is affect. Sentience and phenomenal consciousness cannot both be experience if phenomenal consciousness excludes affect but sentience includes affect, as claimed. Again, the solution to this logical contradiction is to adopt the standard terminology of Table 1.

2. Counterevidence. Although M\&P present considerable convincing evidence that insects have both sentience and sophisticated cognition and learning, they do not include the experimental evidence against these abilities. This counterevidence was presented in the article by Abramson \& Wells (2018), "An inconvenient truth: Some neglected issues in invertebrate learning," which M\&P only cite in passing. Abramson \& Wells report that the learning abilities of bees neurobiologically and behaviorally among the most complex insects - are sometimes more specific and less flexible than those of vertebrates. Bees can become confused when either the spatial location or the target cue for a learned food source is changed experimentally, even modestly (cap-pushing test of Abramson et al., 2016; moved reward site of Sanderson et al., 2013; changed target of Abramson, 1990). These findings are relevant because M\&P urge against using deflationary (simpler) cognitive explanations of invertebrate behaviors when more complex explanations are applied to vertebrates who perform the same behaviors. It is therefore potentially devastating when an invertebrate performs worse, opening the door for deflationary accounts that claim bees are not sentient but just take unconscious shortcuts to solve problems.

Our point is that this valid counterevidence should not be ignored. We do not deny that the preponderance of the evidence suggests that insects are conscious. We found two studies that showed insect consciousness to be especially convincing (Feinberg and Mallatt, 2018, pp. 6062): first, the study by Fauria et al. (2000) where bees learned a complex two-part target, indicating that they form and recall mental images; and second, the study by Perry et al. (2016) where a pre-taste of a sugar reward made bees more likely to interpret ambiguous cues as leading to the reward - a form of optimism. These bees passed the judgement-bias test, a standard way of evaluating whether vertebrates have affective consciousness.

3. Why just arthropods and cephalopods? A strength of M\&P's analysis is that they attribute sentience and moral status only to arthropods and cephalopods among the non-vertebrates. Recognizing which organisms are sentient is difficult. Levy (2020) acknowledges this difficulty, suggesting that we should not even try but should instead assign moral status to cognition, which is easier to recognize. (This is rejected by M\&P and Vonk, 2020.) More relevantly, Figdor (2020) and Veit \& Huebner (2020) acknowledge the difficulty by asking why M\&P draw the sentient line at these particular invertebrates rather than considering whether plants, bacteria, and all living things are sentient. Our own work on which organisms have consciousness (Feinberg and Mallatt, 2016, 2018, 2019, 2020) suggests that M\&P drew the line at the right place.

We derived which organisms are conscious from two logical (and ultimately testable) assumptions. To identify the organisms with affective consciousness (Table 1), we assumed that emotions are revealed by the capacity for global operant learning (extensive learning from experience). We assumed this because this kind of learning provides double evidence of 
emotions: (1) the initial attraction to a reward (or aversion to a punishment); and (2) the recall of the learned reward (or punishment) to motivate behaviors. Our criterion of global operant learning is similar to the Unlimited Associative Learning idea of Bronfman et al. (2016) and Ginsburg and Jablonka (2019).

To identify the organisms with image-based consciousness (Table 1) - mental simulations of the sensed world - we assumed that any organism that constructs multisensory maps of the external environment and of its internal body experiences these maps consciously as mental reference-images for acting in the world. These maps are built from many senses and are detailed enough that they can aid free-living survival, growth, and reproduction in the hostile world capacities far beyond what any known Al or robots have.

If we apply these two assumptions, the only sentient organisms turn out to be the mobile, complex-brained clades of animals, namely, all vertebrates, arthropods, and cephalopods. We documented both the high-capacity operant learning of these animals and the mapped neural pathways to their brains in our first book (Feinberg and Mallatt, 2016), then added more documentation in our second (Feinberg and Mallatt, 2018). This reinforces M\&P's attribution of sentience and moral status to just these three animal clades and not to other living organisms.

Veit \& Huebner (2020), Reber (2016), and others do not think it is possible to draw such a definite boundary between sentient and nonsentient animals. Yet there is a definite anatomic boundary (rapid evolution of complex sensory organs and brains) and a definite historical jump as well. The fossil record shows that the definitive characteristics of consciousness (high mobility as well as complex sensory organs) evolved in the first arthropods and vertebrates during a geologically short time in the Cambrian explosion of animal diversity between 550 and 520 million years ago (Plotnick et al., 2010; Trestman, 2013; Feinberg and Mallatt, 2016; Godfrey-Smith, 2016). We encourage the more philosophical treatments of animal sentience to recognize this often-presented and well-established leap, which also supports the conclusions of M\&P.

\section{References}

Abramson, C. I. (1990). Invertebrate learning: A laboratory manual and source book. American Psychological Association.

Abramson, C. I., \& Wells, H. (2018). An inconvenient truth: Some neglected issues in invertebrate learning. Perspectives on Behavior Science, 41(2), 395-416.

Abramson, C. I., Dinges, C. W., \& Wells, H. (2016). Operant conditioning in honey bees (Apis mellifera L.): The cap pushing response. PLoS One, 11(9), e0162347.

Adolphs, R., \& Anderson, D. J. (2018). The neuroscience of emotion: A new synthesis. Princeton. Balcombe, J. (2020). Intuition and the invertebrate dogma. Animal Sentience 29(9).

Block, N. (1995). On a confusion about a function of consciousness. Behavioral \& Brain Sciences, 18(2), 227-247.

Bronfman, Z. Z., Ginsburg, S., \& Jablonka, E. (2016). The transition to minimal consciousness through the evolution of associative learning. Frontiers in Psychology, 7, 1954.

Browning, H., \& Veit, W. (2020). Improving invertebrate welfare. Animal Sentience 29(4).

Cammaerts, M-C. (2020). Invertebrates should be given ethical consideration. Animal Sentience 29(6). 
Carruthers, P. (2018). Valence and value. Philosophy and Phenomenological Research, 97(3), 658-680.

Elwood, R. W. (2020). Do arthropods respond to noxious stimuli purely by reflex? Animal Sentience 29(10).

Fauria, K., Colborn, M., \& Collett, T. S. (2000). The binding of visual patterns in bumblebees. Current Biology, 10(15), 935-938.

Feinberg, T. E., \& Mallatt, J. M. (2016). The ancient origins of consciousness: How the brain created experience. MIT Press.

Feinberg, T. E., \& Mallatt, J. M. (2018). Consciousness demystified. MIT Press.

Feinberg, T. E., \& Mallatt, J. M. (2019). Subjectivity "demystified": Neurobiology, evolution, and the explanatory gap. Frontiers in Psychology, 10, 1686.

Feinberg, T. E., \& Mallatt, J. M. (2020). Phenomenal consciousness and emergence: Eliminating the explanatory gap. Frontiers in Psychology, 11, 1041.

Figdor, C. (2020). Relationship between cognition and moral status needs overhaul. Animal Sentience 29(3).

Ginsburg, S., \& Jablonka, E. (2019). The evolution of the sensitive soul: Learning and the origins of consciousness. MIT Press.

Godfrey-Smith, P. (2016). Animal evolution and the origins of experience. In How biology shapes philosophy: New foundations for naturalism, ed. D. L. Smith, 51-71. Cambridge University Press.

Levy, N. (2020). It might not matter very much whether insects are conscious. Animal Sentience 29(2).

Mellor, D. J. (2019). Welfare-aligned sentience: Enhanced capacities to experience, interact, anticipate, choose and survive. Animals, 9(7), 440.

Mikhalevich, I. \& Powell, R. (2020). Minds without spines: Evolutionary inclusive animal ethics. Animal Sentience 29(1).

Perry, C.J., Baciadonna, L., \& Chittka, L. (2016). Unexpected rewards induce dopamine dependent positive emotion-like state changes in bumblebees. Science, 353(6307), 15291531.

Plotnick, R. E., Dornbos, S. Q., \& Chen, J. (2010). Information landscapes and sensory ecology of the Cambrian Radiation. Paleobiology, 36(2), 303-317.

Reber, A. S. (2016). Caterpillars, consciousness and the origins of mind. Animal Sentience 11(1).

Sanderson, C. E., Cook, P., Hill, P. S., Orozco, B. S., Abramson, C. I., \& Wells, H. (2013). Nectar quality perception by honey bees (Apis mellifera ligustica). Journal of Comparative Psychology, 127(4), 341.

Soryl, A. (2020). Invertebrate welfare in the wild. Animal Sentience 29(7).

Trestman, M. (2013). The Cambrian explosion and the origins of embodied cognition. Biological Theory, 8(1), 80-92.

Veit, W., \& Huebner, B. (2020). Drawing the boundaries of animal sentience. Animal Sentience 29(13).

Vonk, J. (2020). No room for speciesism in welfare considerations. Animal Sentience 29(5). 